\title{
Document Type Profiles in Nature, Science, and PNAS: Journal and Country Level
}

\author{
Jielan Ding ${ }^{1,2}$, Per Ahlgren 3 , Liying Yang ${ }^{1 \dagger} \&$ Ting Yue ${ }^{1}$ \\ ${ }^{1}$ National Science Library, Chinese Academy of Sciences, Beijing 100190, China \\ ${ }^{2}$ University of Chinese Academy of Sciences, Beijing 100049, China \\ ${ }^{3}$ School of Education and Communication in Engineering Sciences (ECE), KTH Royal \\ Institute of Technology, Stockholm 100 44, Sweden
}

Citation: Jielan Ding, Per Ahlgren, Liying Yang \& Ting Yue (2016). Document Type Profiles in Nature, Science, and $P N A S$ : Journal and Country Level.

Received: Jun. 12, 2016 Revised: Aug. 11, 2016 Accepted: Aug. 17, 2016 the United States (PNAS) with regard to two levels: journal and country.

Design/methodology/approach: Using relative values based on fractional counting, we investigate the distribution of publications across document types at both the journal and country level, and we use (cosine) document type profile similarity values to compare pairs of publication years within countries.

Findings: Nature and Science mainly publish Editorial Material, Article, News Item and Letter, whereas the publications of PNAS are heavily concentrated on Article. The shares of Article for Nature and Science are decreasing slightly from 1999 to 2014, while the corresponding shares of Editorial Material are increasing. Most studied countries focus on Article and Letter in Nature, but on Letter in Science and PNAS. The document type profiles of some of the studied countries change to a relatively large extent over publication years.

Research limitations: The main limitation of this research concerns the Web of Science classification of publications into document types. Since the analysis of the paper is based on document types of Web of Science, the classification in question is not free from errors, and the accuracy of the analysis might be affected.

Practical implications: Results show that Nature and Science are quite diversified with regard to document types. In bibliometric assessments, where publications in Nature and Science play a role, other document types than Article and Review might therefore be taken into account.

Originality/value: Results highlight the importance of other document types than Article and Review in Nature and Science. Large differences are also found when comparing the country document type profiles of the three journals with the corresponding profiles in all Web of Science journals.

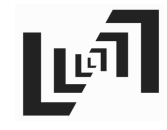

JDIS

Journal of Data and Information Science

Vol. 1 No. 3, 2016

pp 27-41

Corresponding author: Liying Yang (E-mail: yangly@mail.las.ac.cn).
DOI: $10.20309 /$ jdis. 201618 http://www.jdis.org 


\section{Research Paper}

Keywords Country; Document type profile; Nature; Science; Proceedings of the National Academy of Sciences of the United States of America (PNAS)

\section{Introduction}

In bibliometric assessments, Article and Review are document types of scientific communication that are usually taken into account, while other types, like Editorial Material, are often excluded. Earlier studies have dealt with the issue of which document types should be included in the assessments. Some of these studies treat a certain document type and argue that the type should be included in the assessments, e.g. Book Review (Zuccala \& van Leeuwen, 2011), Editorial Material (van Leeuwen et al., 2013), and Proceedings Paper (Michels \& Fu, 2014). Research that concerns document type classification problems in Web of Science (WoS) has been done, where the corresponding studies treat document types such as Research Letter (van Leeuwen et al., 2007) and Proceedings Paper (Michels \& Fu, 2014), and the validity of the used classification (Harzing, 2010; 2013; van Leeuwen et al., 2007). Lewison (2009) investigated the usage of document type as an indicator in research output assessment, whereas Zhang, Rousseau, \& Glänzel (2011) studied country profiles in relation to document types. Citation impact of document types has been studied (Braun, Glänzel, \& Schubert, 1989b; Campanario et al., 2011; Frandsen, 2008; Garfield, 1987; van Leeuwen et al., 1999; Rousseau, 2009; Sigogneau, 2000), as well as document types used in social media (Haustein, Costas, \& Larivière, 2015).

Research on document types has mainly focused on all journals covered by WoS. In this study, we restrict our attention to the three journals Nature, Science, and Proceedings of the National Academy of Sciences of the United States (PNAS). In view of the prestige these three journals have within the scientific community, and the important role they play in various research assessments, we believe it is of importance to study them separately. Earlier research on Nature, Science, and PNAS has compared their internationality (e.g. Kaneiwa et al., 1988), their publication patterns and citation impact (e.g. Braun, Glänzel, \& Schubert, 1989a; Wang et al., 2008), and their dynamic usage history (e.g. Wang et al., 2014). However, in this study, we examine the document types of the three journals using two levels of analysis: journal and country.

\section{Data and Methods}

A total of 35,816 publications, appearing in the three journals, were retrieved from WoS on November 15, 2015. News Item is one of the main document types of Nature and Science, but it was not formally indexed in WoS until 1996. In view

Journal of Data and of this, we do not use publications published before 1996 in order to avoid Information Science comparisons, between years, which might be affected by the absence/presence of 
News Item publications in the journals. We use a 5-year time gap, year 1999 as the start year and year 2014 as the end year.

The number of publications of each journal for the four years is shown in Table 1. At the journal level of the study, we used all publications appearing in the three journals. There exist, however, publications without addresses in our dataset. Therefore, at the country level of the study, we only used all publications with addresses appearing in the three journals. At this level, we made use of address fractionalization. Clearly, due to co-authorship, a publication might involve more than one country among its addresses. If, for instance, a publication has two USA addresses and one France address, USA contributes 2/3 and France 1/3 to the publication.

Table 1. Number of publications of the journals.

\begin{tabular}{|c|c|c|c|c|c|c|c|c|}
\hline \multirow{2}{*}{ Journal } & \multicolumn{2}{|c|}{1999} & \multicolumn{2}{|c|}{2004} & \multicolumn{2}{|c|}{2009} & \multicolumn{2}{|c|}{2014} \\
\hline & All & With add. & All & With add. & All & With add. & All & With add \\
\hline Nature & 3,000 & 2,014 & 2,603 & 1,766 & 2,544 & 1,666 & 2,561 & 1,708 \\
\hline Science & 2,726 & 1,729 & 2,682 & 1,711 & 2,516 & 1,724 & 2,602 & 1,631 \\
\hline$P N A S$ & 2,827 & 2,759 & 3,336 & 3,208 & 4,220 & 4,078 & 4,199 & 3,998 \\
\hline
\end{tabular}

Table 2 reports the 10 most productive countries, regarding fractional publication output, in the three journals in the publication year 2014. The countries are ordered descending according to the total number of publication fractions in the three journals. In addition to the top 10 countries, we included the other (relative to People's Republic of China) four BRICS countries as cases for comparison, i.e Brazil, Russia, India, and South Africa.

Table 2. Number of publication fractions of the 10 most productive countries and four BRICS countries in year 2014 .

\begin{tabular}{lrrrrrrr}
\hline \multirow{2}{*}{ Countries } & \multicolumn{2}{c}{ Number of publication fractions } & & \multicolumn{3}{c}{ Rank } \\
\cline { 2 - 3 } \cline { 6 - 8 } & Nature & Science & PNAS & & Nature & Science & PNAS \\
\hline USA & 807.4 & 872.1 & $2,261.8$ & & 1 & 1 & 1 \\
UK & 208.8 & 159.8 & 259.1 & & 2 & 2 & 2 \\
Germany & 93.2 & 87.2 & 195.5 & & 3 & 3 & 3 \\
P. R. China & 61.1 & 46.9 & 169.9 & & 6 & 6 & 4 \\
France & 65.5 & 49.8 & 129.3 & & 5 & 4 & 6 \\
Canada & 49.7 & 48.3 & 116.7 & & 7 & 5 & 7 \\
Japan & 31.4 & 42.6 & 139.4 & & 10 & 7 & 5 \\
Australia & 68.8 & 41.0 & 73.8 & & 4 & 8 & 8 \\
Switzerland & 48.6 & 38.3 & 64.2 & & 8 & 9 & 10 \\
Netherlands & 34.7 & 36.1 & 69.9 & & 9 & 10 & 9 \\
Brazil & 8.8 & 9.4 & 13.1 & & 19 & 18 & 24 \\
India & 6.0 & 6.3 & 12.2 & & 25 & 21 & 25 \\
Russia & 7.3 & 3.4 & 7.2 & & 21 & 28 & 30 \\
South Africa & 4.3 & 5.5 & 5.2 & & 30 & 23 & 32 \\
\hline
\end{tabular}

Journal of Data and Information Science 


\section{Research Paper}

In the bibliographic records of WoS, the document type of each publication is recorded. Based on this information, we calculated, for a given journal, a given document type and a given year the number of publications for the combination. In order to map the document type profile for each of the three journals across time, the raw numbers were used to obtain the relative contribution of publications to each document type, and for each considered year, with respect to all publications published in the journal.

For journal $i$, document type $j$, and publication year $y$, we define $P_{i j y}$ as the number of publications of journal $i$ of document type $j$ in year $y$, and $R_{i j y}$ as the percentage of publications of document type $j$ of journal $i$ in year $y$ relative to the total number of publications of journal $i$ in year $y$. Formally:

$$
R_{i j y}=P_{i j y} / P_{i y} \times 100,
$$

where $P_{i v}$ is the total number of publications of journal $i$ in year $y$.

Then, for journal $i$ in year $y$, the (relative) document type distribution is represented by the vector $D_{i y}$, defined as in Equation (2):

$$
D_{i y}=\left(R_{i l y}, R_{i 2 y}, \ldots, R_{i n y}\right),
$$

where $n$ is the number of document types of journal $i$ in year $y$.

For a country $c$, we define $P_{i j, y c}$ as the number of $c$ publication fractions in journal $i$ of document type $j$ in year $y$, and $R_{i j y c}$ as the percentage of $c$ publication fractions in journal $i$ of document type $j$ in year $y$ relative to $P_{i j y}^{\prime}$, the number of publications, with at least one address, in journal $i$ of document type $j$ in year $y$. Formally:

$$
R_{i j y c}=P_{i j y c} / P_{i j y}^{\prime} \times 100 .
$$

Since fractionalization is used, the sum of the percentages across all countries in journal $i$, with regard to document type $j$ and in year $y$, is equal to 100 . For country $c$ in journal $i$ in year $y$, the (relative) document type distribution is represented by the vector $D_{i y c}$, defined as Equation (4):

$$
D_{i y c}=\left(R_{i l y c}, R_{i z y o} \ldots, R_{i n y c}\right),
$$

where $n$ is the number of document types of journal $i$ in year $y$.

By application of the cosine measure, we obtained similarities, for each journal, for pairs of years within a country. The similarity between years $y$ and $y+5$ for country $c$ in journal $i$ is defined as Equation (5):

Journal of Data and

$$
S_{i y(y+5) c}=\frac{\sum_{j=1}^{n} R_{i j y c} \times R_{i j(y+5) c}}{\sqrt{\sum_{j=1}^{n}\left(R_{i j y c}\right)^{2}} \times \sqrt{\sum_{j=1}^{n}\left(R_{i j(y+5) c}\right)^{2}}} .
$$




\section{Results}

\subsection{Document Type Profiles at the Journal Level}

Figures 1-3 show the document type profiles of Nature, Science, and PNAS, respectively, for the years 1999, 2004, 2009, and 2014 (the percentages in boxes apply to year 2014; the underlying data is shown in Tables A1, A2, and A3 in Appendix). Notice that the bars across the document types, for a given journal and a given year, correspond to a vector of the type defined in Equation (2). From Figures 1 and 2, it is clear that Nature and Science have quite diversified document type profiles, where the types are foremost Article, Editorial Material, News Item, and Letter. For PNAS, however, its publications are heavily concentrated on the type Article, regardless of year. This outcome reflects the difference in aims and scope of Nature, Science, and PNAS. Nature and Science are not only research journals but also scientific news magazines, whereas $P N A S$ is strongly oriented towards research.

Comparing the changes in percentages for the two types Article and Editorial Material across different years in Nature and Science, we find that the percentages of Article are decreasing slightly from 1999 to 2014, while the percentages for Editorial Material are increasing (Figures 1 and 2, respectively). Such material represents scientific news or opinions of experts on scientific news. This outcome

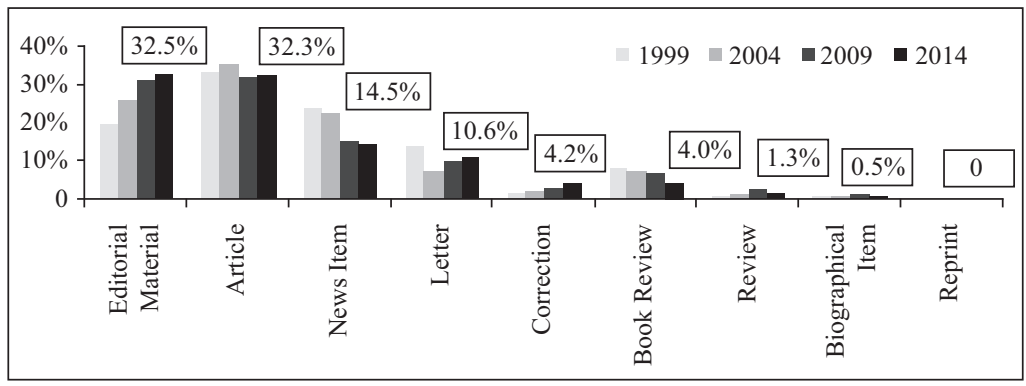

Figure 1. Document type profiles in Nature in years 1999, 2004, 2009, and 2014.

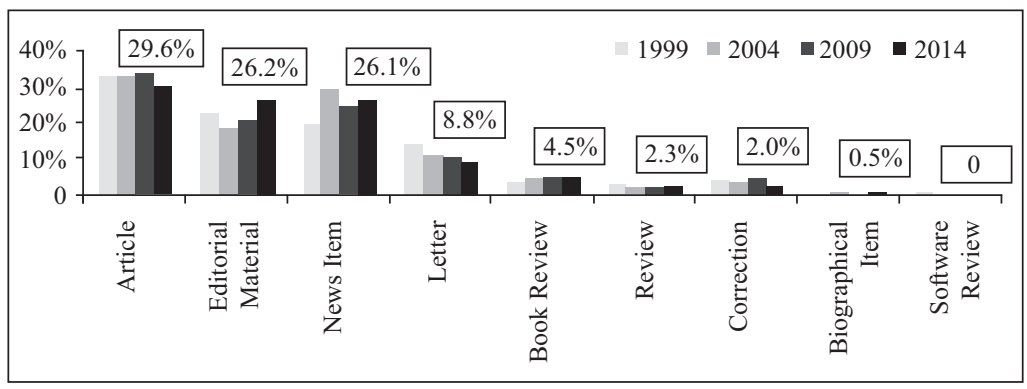

Figure 2. Document type profiles in Science in years 1999, 2004, 2009, and 2014.

Journal of Data and Information Science 


\section{Research Paper}

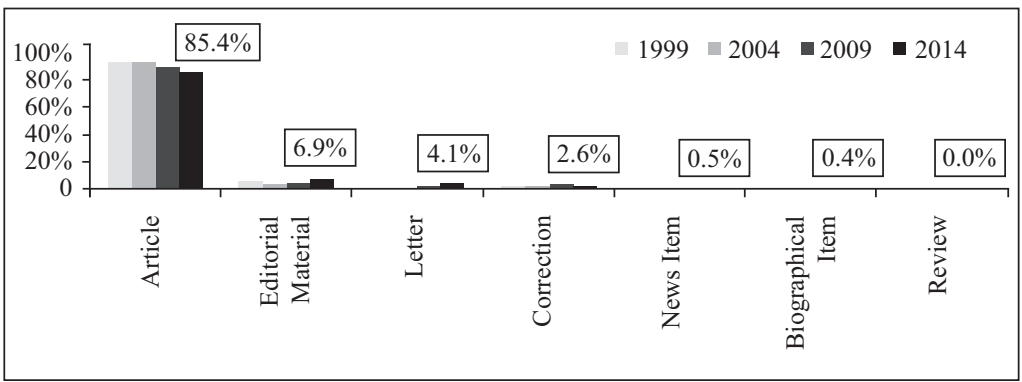

Figure 3. Document type profiles in PNAS in years $1999,2004,2009$, and 2014 . Note that $0.0 \%$ is the result rounded to the nearest tenth.

indicates that reporting news or expert opinions on important research tends to be increasingly important. Notice that for Nature, the absolute numbers of publications decrease from 1999 to 2009 (Table 1). Thus, the increasing percentages for Editorial Material are consistent with the assumption that the absolute numbers of Editorial Material are constant. However, the latter is not the case: these absolute numbers strictly increase across the four considered years (Table A1).

\subsection{Document Type Profiles at the Country Level}

The publication volumes of the document types Review, Biographical Item, and Reprint in Nature in year 2014 are small (Tables A1, A2, and A3 in the Appendix). Therefore, these three document types are not taken into account at the country level. Moreover, the types Correction and News Item were also excluded, since publications of these types often lack addresses in our Nature data. Also for Science and $P N A S$, and for similar reasons as in the Nature case, some document types were excluded. For all three journals, and with respect to comparison between countries, we restrict the result presentation to the latest publication year in the study, 2014.

Figures 4-6 visualize for the three journals, in the form of radar plots, the 2014 document type profiles of the countries. Notice that a radar plot corresponds to a vector of the type defined in Equation (4). Sample observations:

\section{- Nature}

USA exhibits a relative balanced profile in Nature, with similar emphasis on Article, Book Review, and Editorial Material (50\%-56\% of the total publication fraction output across the countries for these types) and with less emphasis on Letter.

Several countries focus strongly on Article. The outcomes for Switzerland and Germany suggest a rhombus document type profile with great emphasis on Article. France, Japan, and Russia show a

Journal of Data and triangle document type profile with more emphasis on Article and less emphasis on Letter and Editorial Material. 
Several countries focus on Letter rather than other document types. The outcomes for People's Republic of China and Australia suggest a rhombus document type profile with great emphasis on Letter. India and Brazil have a triangle document type profile with more emphasis on Letter than Article and Editorial Material.

- Science

$>$ As in the Nature case, USA has a fairly even profile, and stands for the largest percentages $(47 \%-57 \%$ of the total publication fraction output across the countries for all four included types). Switzerland has a relatively balanced profile with similar emphasis on Article, Editorial Material, and Book Review.

Most studied countries focus strongly on the type Letter. Canada, Australia, People's Republic of China, and Brazil have a rhombus document type profile with strong emphasis on Letter. India and South Africa have a triangle document type profile with the strongest emphasis on Letter.

- PNAS

Germany and UK have an "equilateral triangle" profile, which suggests a balanced structure, with similar emphasis on Article, Editorial Material, and Letter.

$>$ USA and Canada have an "isosceles triangle" structure with most emphasis on Editorial Material, and similar emphasis on Article and Letter. Notice that USA accounts for about $70 \%$ of the total publication fraction output across the countries for the type Editorial Material.

Most studied countries focus strongly on Letter, like France, People's Republic of China, Australia, and the Netherlands.

Table $3(4,5)$ gives the cosine similarity values for pairs of years within the 14 countries in Nature (Science, PNAS). In all three figures, values below 0.8 are marked in grey. For Nature and Science, the document type profiles of most countries change only slightly between different years. With regard to PNAS, for a majority of the countries, the similarity values for the document type profiles for the years 2004 and 2009 are generally much lower compared to the other year pairs. The profiles of some BRICS countries change to a relatively large extent. For instance, the profiles of India and South Africa (2004 vs. 2009; 2009 vs. 2014) regarding PNAS (Table 5). The radar plots of Figure 7 visualize the 2004, 2009 and 2014 document type profiles of these two countries in PNAS. Clearly, the profile structures are quite different across the three years for both India and South Africa, which is reflected by the corresponding low cosine values.

Journal of Data and Information Science 


\section{Research Paper}

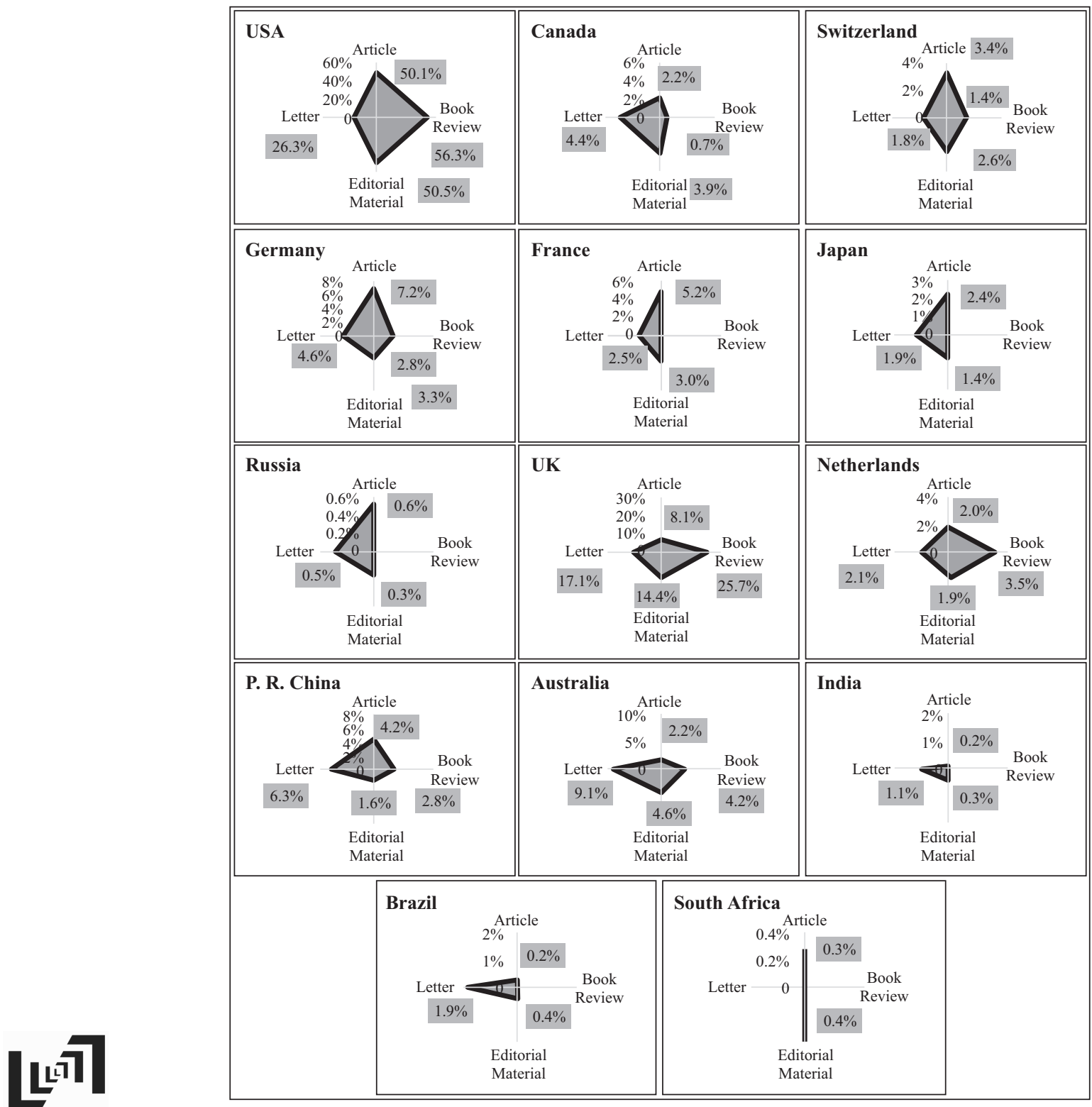

Figure 4. Radar plots representing the country document type profiles in Nature in year 2014.

Journal of Data and Information Science 


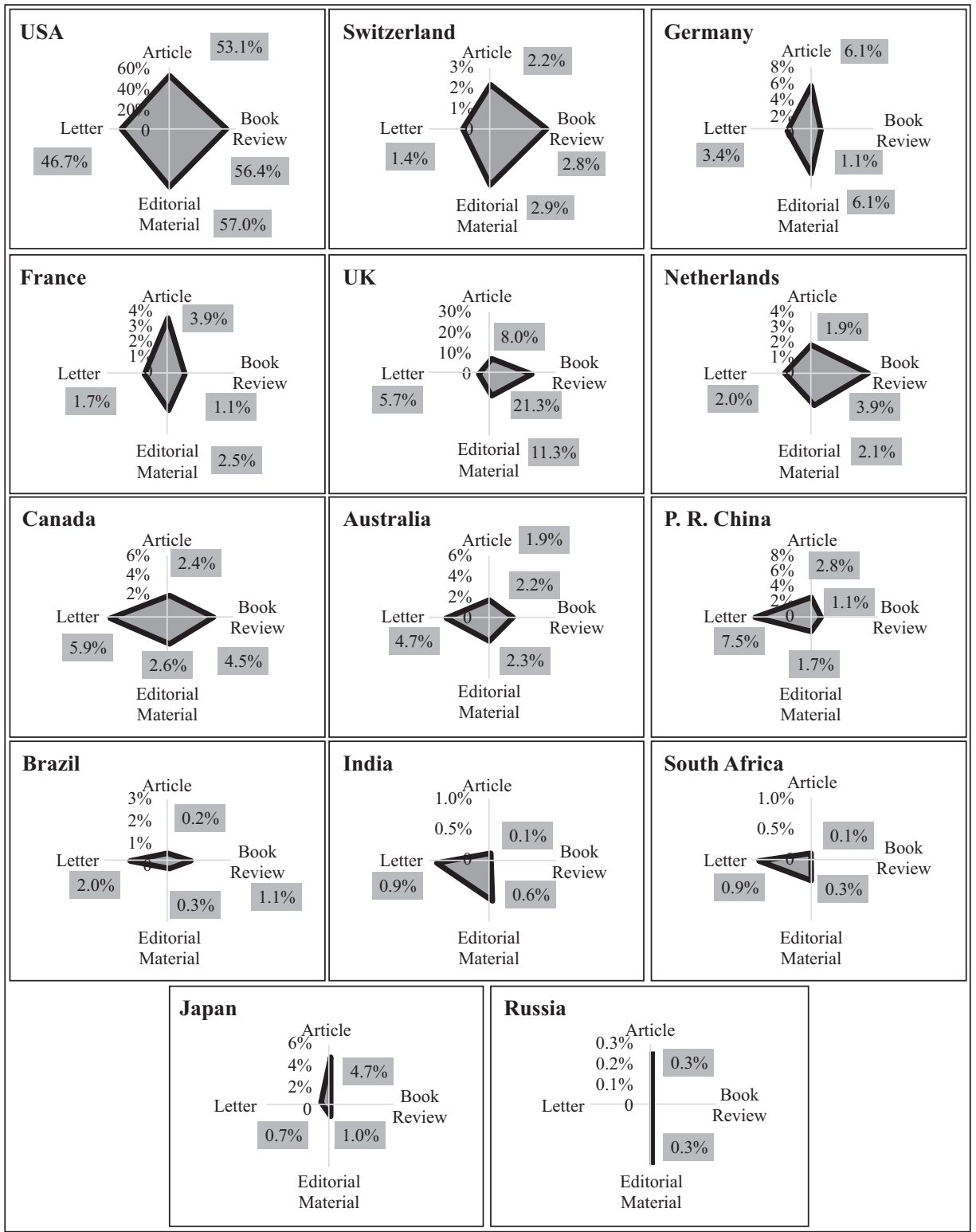

Figure 5. Radar plots representing country document type profiles in Science in year 2014.

Journal of Data and Information Science 


\section{Research Paper}

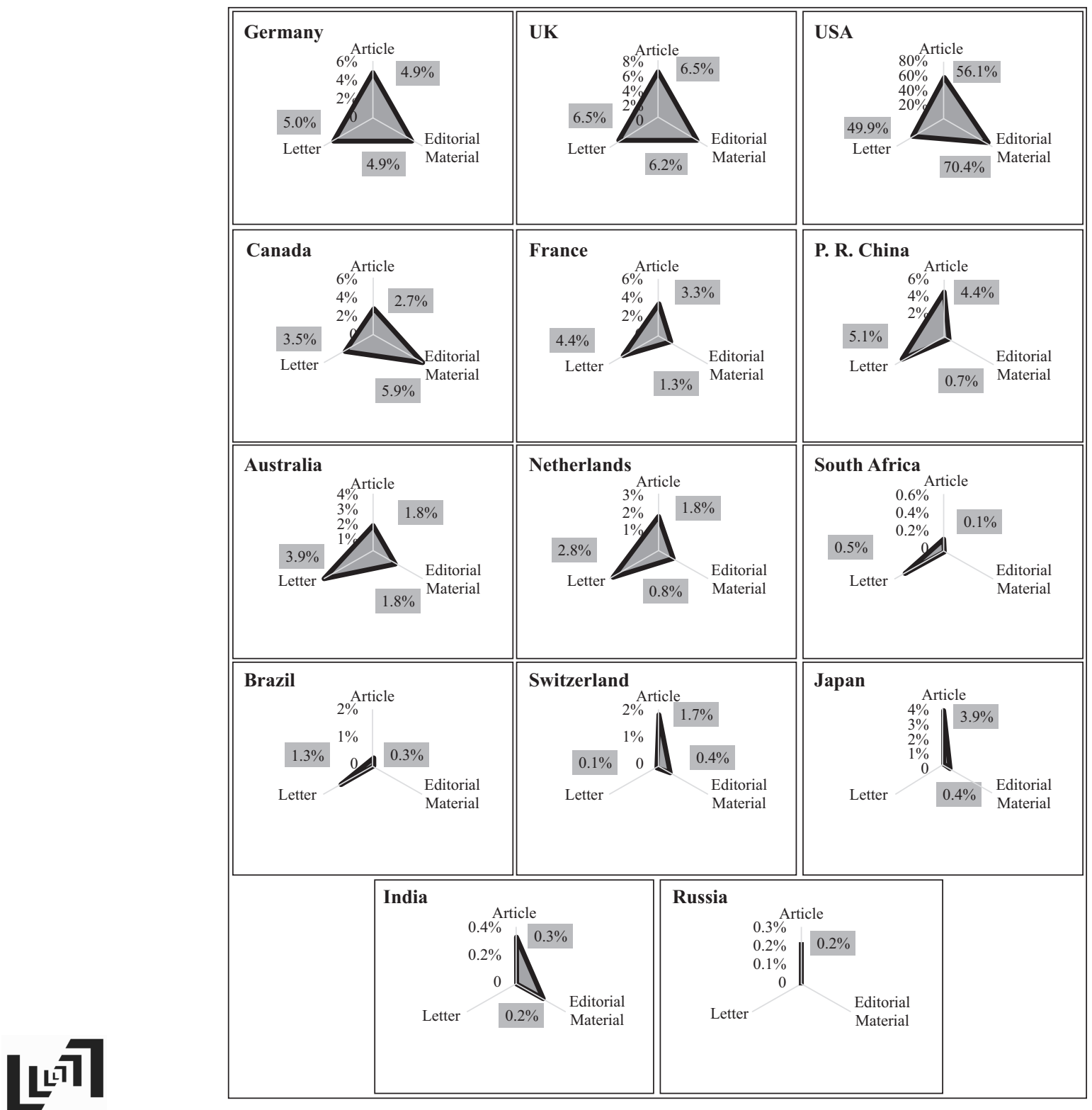

Figure 6. Radar plots representing country document type profiles in PNAS in year 2014.

Journal of Data and Information Science 
Document Type Profiles in Nature, Science, and PNAS: Journal and Country Level

Table 3. Nature: Similarity values for country document type profiles for pairs of years.

\begin{tabular}{lccc||lccc}
\hline Countries & $1999 v s .2004$ & 2004 vs. 2009 & 2009vs. 2014 & Countries & 1999 vs. 2004 & 2004 vs. 2009 2009vs. 2014 \\
\hline Australia & 0.695 & 0.689 & 0.973 & Netherlands & 0.991 & 0.739 & 0.980 \\
Brazil & 0.944 & 0.989 & 0.996 & P. R. China & 0.667 & 0.508 & 0.887 \\
Canada & 0.920 & 0.950 & 0.919 & Russia & 0.741 & 0.943 & 0.844 \\
France & 0.987 & 0.946 & 0.912 & South Africa & 0.233 & 0.998 & 0.463 \\
Germany & 0.968 & 0.965 & 0.986 & Switzerland & 0.976 & 0.926 & 0.897 \\
India & 0.624 & 0.984 & 0.957 & UK & 0.992 & 0.995 & 0.989 \\
Japan & 0.936 & 0.989 & 0.844 & USA & 1.000 & 0.999 & 0.979 \\
\hline
\end{tabular}

Table 4. Science: Similarity values for country document type profiles for pairs of years.

\begin{tabular}{lccc||lccc}
\hline Countries & $1999 v s .2004$ & 2004 vs. 2009 & 2009vs. 2014 & Countries & 1999vs. 2004 2004 vs. 2009 2009vs. 2014 \\
\hline Australia & 0.647 & 0.961 & 0.983 & Netherlands & 0.897 & 0.888 & 0.953 \\
Brazil & 0.964 & 0.950 & 0.815 & P. R. China & 0.923 & 0.933 & 0.810 \\
Canada & 0.821 & 0.939 & 0.903 & Russia & 0.496 & 0.945 & 0.603 \\
France & 0.937 & 0.970 & 0.961 & South Africa & 0.952 & 0.955 & 0.989 \\
Germany & 0.926 & 0.970 & 0.991 & Switzerland & 0.911 & 0.950 & 0.906 \\
India & 0.642 & 0.545 & 0.957 & UK & 0.985 & 0.992 & 0.975 \\
Japan & 0.994 & 0.907 & 0.911 & USA & 0.995 & 0.998 & 0.995 \\
\hline
\end{tabular}

Table 5. PNAS: Similarity values for country document type profiles for pairs of years.

\begin{tabular}{|c|c|c|c|c|c|c|c|}
\hline Countries & 99 vs. 2004 & 2004 vs. 2009 & 2009 vs. 2014 & Countries & 1999 vs. 2004 & 2004 vs. 2009 & 2009 vs. 2014 \\
\hline Australia & 0.601 & 0.711 & 0.973 & Netherlands & 0.773 & 0.371 & 0.968 \\
\hline Brazil & 1.000 & 0.941 & 0.540 & P. R. China & 0.133 & 0.993 & 0.660 \\
\hline Canada & 0.993 & 0.739 & 0.969 & Russia & 1.000 & 1.000 & 1.000 \\
\hline France & 0.968 & 0.639 & 0.996 & South Africa & 1.000 & 0.169 & 0.042 \\
\hline Germany & 0.992 & 0.660 & 0.979 & Switzerland & 0.998 & 0.736 & 0.721 \\
\hline India & 1.000 & 0.138 & 0.114 & UK & 0.948 & 0.730 & 0.988 \\
\hline Japan & 0.987 & 0.896 & 0.915 & USA & 1.000 & 0.879 & 1.000 \\
\hline
\end{tabular}

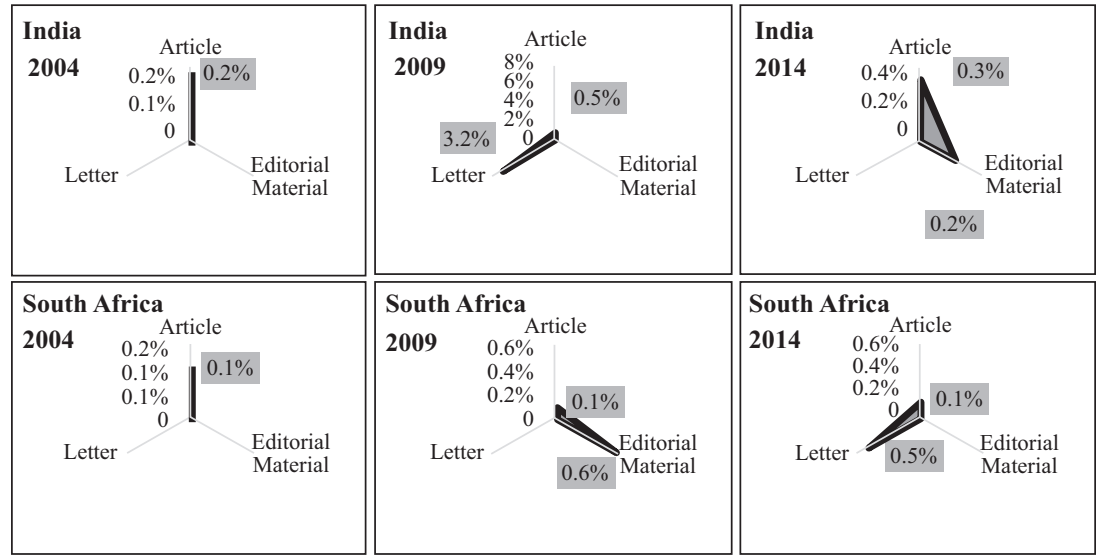

Figure 7. Radar plots representing document type profiles of India and South Africa in PNAS in years 2004, 2009 , and 2014.

Journal of Data and Information Science 


\section{Research Paper}

\section{Discussion and Conclusion}

In this study, we have treated the document type profiles in the three journals Nature, Science and PNAS. We analyzed the profiles at two levels, journal and country. The results for the journal level show that Nature and Science mainly publish Editorial Material, Article, News Item and Letter. The publications of $P N A S$, though, are heavily concentrated on the type Article, regardless of year. This outcome mirrors the difference in aims and scope of the journals. Nature and Science aim at publishing remarkable scientific research and important scientific news related to such research. PNAS is a very academic journal, which almost exclusively publishes high-level frontier research. Interestingly, the percentages for the type Article for Nature and Science are decreasing slightly from 1999 to 2014, while the corresponding percentages for Editorial Material are increasing. Van Leeuwen (2013) explored the possibilities to include editorial materials in research assessment procedures. With regard to citation impact, the difference between articles and editorial materials turned out to be relatively small. Partly based on this outcome, the authors concluded that some editorial materials can be included in research assessment procedures.

At the country level, the results show, with regard to year 2014, that most studied countries focus on Article and Letter in Nature, but on Letter in Science and PNAS. The BRICS countries, except People's Republic of China, have rather unbalanced document type profiles, and this is the case also for Japan. The publication output of Brazil, Russia, India, and South Africa in the three journals is small, a fact that might explain the unbalanced profiles of these countries. For the comparison of profiles between different years, there are BRICS countries for which the profiles change to a quite large extent.

Large differences are found when comparing the country document type profiles of the three journals with the corresponding profiles in all WoS journals (Zhang, Rousseau, \& Glänzel, 2011). For example, with respect to all WoS journals, it was shown that People's Republic of China published almost exclusively documents of the type Article, whereas the publishing emphasis of China in Nature, Science, and $P N A S$ is on Letter. A similar pattern can be observed for several other countries of this study. This suggests that the country document type profiles of Nature, Science, and PNAS are different from the corresponding profiles of other WoS journals.

For future research, we would like to compare the citation impact of different document types in Nature, Science, and PNAS with their impact across all WoS journals.

Journal of Data and Information Science 


\section{Acknowledgements}

We thank Ronald Rousseau and RainerFrietsch for valuable comments. This work is supported by the National Natural Science Foundation of China (Grant No.: L1524037).

\section{Author Contributions}

J.L. Ding (dingjielan@mail.las.ac.cn) performed the research, designed the research framework, collected and analyzed the data, wrote the first draft and revised the paper. P. Ahlgren (perahl@ kth.se) designed the research framework, collected and analyzed the data, wrote and revised the manuscript. L.Y. Yang (yangly@mail.las.ac.cn, corresponding author) put forward the research idea, planned and designed the outline and contributed to the writing of the paper. T. Yue (yuet@ mail.las.ac.cn) joined discussion of the findings and contributed to the writing of the paper.

\section{References}

Braun, T., Glänzel, W., \& Schubert A. (1989a). National publication patterns and citation impact in the multidisciplinary journals Nature and Science. Scientometrics, 17(1-2), 11-14.

Braun, T., Glänzel, W., \& Schubert, A. (1989b). Some data on the distribution of journal publication types in the Science Citation Index database. Scientometrics, 15(5-6), 325-330.

Campanario, J.M., Carretero, J., Marangon, V., Molina, A., \& Ros, G. (2011). Effect on the journal impact factor of the number and document type of citing records: a wide-scale study. Scientometrics, 87, 75-84.

Frandsen, T.F. (2008). On the ratio of citable versus non-citable items in economics journals. Scientometrics, 74, 439-451.

Garfield, E. (1987). Why are the impacts of the leading medical journals so similar and yet so different? Item-by-item audits reveal a diversity of editorial material. Current Contents, 2, $7-13$.

Harzing, A.W. (2010). Working with ISI data: Beware of categorisation problems. Retrieved from http://www.harzing.com/ISI_categories.htm.

Harzing, A.W. (2013). Document categories in the ISI Web of Knowledge: Misunderstanding the social sciences? Scientometrics, 94(1), 23-34.

Haustein, S., Costas, R., \& Larivière, V. (2015). Characterizing social media metrics of scholarly papers: The effect of document properties and collaboration patterns. PloS ONE, 10(3), e0120495.

Kaneiwa, K., Adachi, J., Aoki, M., Masuda, T., Midorikawa, N., Tanimura, A., \& Yamazaki, S. (1988). A comparison between the journals Nature and Science. Scientometrics, 13(3-4), $125-153$.

Lewison, G. (2009). The percentage of reviews in research output: A simple measure of research esteem. Research Evaluation, 18(1), 25-37.

Michels, C., \& Fu, J.Y. (2014). Systematic analysis of coverage and usage of conference proceedings in web of science. Scientometrics, 100(2), 307-327.

Journal of Data and Information Science 


\section{Research Paper}

Rousseau, R. (2009). The most influential editorials. In In Åström, F., Danell, R., \& Larsen, B1, et al. (Eds.) Celebrating Scholarly Communication Studies. A Festschrift for Olle Persson at His $60^{\text {th }}$ Birthday (pp. 47-53). Leuven, Belgium: International Society for Scientometrics and Informetrics.

Sigogneau, A. (2000). An analysis of document types published in journals related to physics: Proceeding publications recorded in the Science Citation Index database. Scientometrics, 47(3), 589-604.

van Leeuwen, T.N., Moed, H.F., \& Reedijk, J. (1999). Critical comments on Institute for Scientific Information impact factors: a sample of inorganic molecular chemistry journals. Journal of Information Science, 25(6), 489-498.

van Leeuwen, T.N., van der Wurff, L.J., \& de Craen, A.J.M. (2007). Classification of "research letters" in general medical journals and its consequences in bibliometric research evaluation processes. Research Evaluation, 16(1), 59-63.

van Leeuwen, T., Costas, R., Calero-Medina, C., \& Visser, M. (2013). The role of editorial material in bibliometric research performance assessments. Scientometrics, 95(2), 817-828.

Wang, Y.H., Fang, C., Sun, S.J., \& Wang, X. (2008). Analysis of Proceedings of the National Academy of Science of the United States (PNAS) (in Chinese). Chinese Journal of Scientific and Technical Periodicals, 29(4), 718-722.

Wang, X.W., Mao, W.L., Xu, S.M., \& Zhang, C.B. (2014). Usage history of scientific literature: Nature metrics and metrics of Nature publications. Scientometrics, 98, 1923-1933.

Zhang, L., Rousseau, R., \& Glänzel, W. (2011). Document-type country profiles. Journal of the American Society for Information Science and Technology, 62(7), 1403-1411.

Zuccala, A., \& van Leeuwen, T.N. (2011). Book reviews in humanities research evaluations. Journal of the American Society of Information Science and Technology, 62, 1979-1991.

\section{(c) $($ () $९$}

This is an open access article licensed under the Creative Commons Attribution-NonCommercialNoDerivs License (http://creativecommons.org/licenses/by-nc-nd/4.0/).

Journal of Data and Information Science 


\section{Appendix A}

Table A1. Nature. Numbers of publications by document type.

\begin{tabular}{lrrrr||rrrr}
\hline \multirow{2}{*}{ Year } & \multicolumn{3}{c||}{ Number of publications } & \multicolumn{4}{c}{ Percentage } \\
\cline { 2 - 8 } Document type & 1999 & 2004 & 2009 & 2014 & 1999 & 2004 & 2009 & 2014 \\
\hline Editorial Material & 574 & 660 & 780 & 833 & $19.1 \%$ & $25.4 \%$ & $30.7 \%$ & $32.5 \%$ \\
Article & 998 & 916 & 800 & 828 & $33.3 \%$ & $35.2 \%$ & $31.4 \%$ & $32.3 \%$ \\
News Item & 709 & 584 & 381 & 371 & $23.6 \%$ & $22.4 \%$ & $15.0 \%$ & $14.5 \%$ \\
Letter & 404 & 183 & 250 & 272 & $13.5 \%$ & $7.0 \%$ & $9.8 \%$ & $10.6 \%$ \\
Correction & 41 & 45 & 78 & 108 & $1.4 \%$ & $1.7 \%$ & $3.1 \%$ & $4.2 \%$ \\
Book Review & 232 & 180 & 169 & 102 & $7.7 \%$ & $6.9 \%$ & $6.6 \%$ & $4.0 \%$ \\
Review & 18 & 21 & 66 & 34 & $0.6 \%$ & $0.8 \%$ & $2.6 \%$ & $1.3 \%$ \\
Biographical Item & 21 & 14 & 19 & 13 & $0.7 \%$ & $0.5 \%$ & $0.7 \%$ & $0.5 \%$ \\
Reprint & 3 & 0 & 1 & 0 & $0.1 \%$ & 0 & $0.0 \%$ & 0 \\
Total & 3,000 & 2,603 & 2,544 & 2,561 & $100.0 \%$ & $100.0 \%$ & $100.0 \%$ & $100.0 \%$ \\
\hline
\end{tabular}

Note. $0.0 \%$ is the result rounded to the nearest tenth.

Table A2. Science. Numbers of publications by document type.

\begin{tabular}{lrrrr||rrrr}
\hline & \multicolumn{1}{c}{ Year } & \multicolumn{3}{c||}{ Number of publications } & \multicolumn{4}{c}{ Percentage } \\
\cline { 2 - 9 } Document type & 1999 & 2004 & 2009 & 2014 & 1999 & 2004 & 2009 & 2014 \\
\hline Article & 893 & 873 & 843 & 769 & $32.8 \%$ & $32.6 \%$ & $33.5 \%$ & $29.6 \%$ \\
Editorial Material & 609 & 490 & 520 & 683 & $22.3 \%$ & $18.3 \%$ & $20.7 \%$ & $26.2 \%$ \\
News Item & 534 & 787 & 611 & 679 & $19.6 \%$ & $29.3 \%$ & $24.3 \%$ & $26.1 \%$ \\
Letter & 388 & 281 & 257 & 230 & $14.2 \%$ & $10.5 \%$ & $10.2 \%$ & $8.8 \%$ \\
Book Review & 93 & 102 & 117 & 117 & $3.4 \%$ & $3.8 \%$ & $4.7 \%$ & $4.5 \%$ \\
Review & 78 & 51 & 54 & 59 & $2.9 \%$ & $1.9 \%$ & $2.1 \%$ & $2.3 \%$ \\
Correction & 100 & 85 & 106 & 52 & $3.7 \%$ & $3.2 \%$ & $4.2 \%$ & $2.0 \%$ \\
Biographical Item & 7 & 12 & 8 & 13 & $0.3 \%$ & $0.4 \%$ & $0.3 \%$ & $0.5 \%$ \\
Software Review & 24 & 1 & 0 & 0 & $0.9 \%$ & $0.0 \%$ & 0 & 0 \\
Total & 2,726 & 2,682 & 2,516 & 2,602 & $100.0 \%$ & $100.0 \%$ & $100.0 \%$ & $100.0 \%$ \\
\hline
\end{tabular}

Note. $0.0 \%$ is the result rounded to the nearest tenth.

Table A3. PNAS. Numbers of publications by document type.

\begin{tabular}{lrrrr||rrrr}
\hline & \multicolumn{3}{c}{ Year } & \multicolumn{3}{c||}{ Number of publications } & \multicolumn{4}{c}{ Percentage } \\
\cline { 2 - 9 } Document type & 1999 & 2004 & 2009 & 2014 & 1999 & 2004 & 2009 & 2014 \\
\hline Article & 2,603 & 3,077 & 3,759 & 3,586 & $92.1 \%$ & $92.2 \%$ & $89.1 \%$ & $85.4 \%$ \\
Editorial Material & 151 & 129 & 181 & 290 & $5.3 \%$ & $3.9 \%$ & $4.3 \%$ & $6.9 \%$ \\
Letter & 0 & 0 & 122 & 174 & 0 & 0 & $2.9 \%$ & $4.1 \%$ \\
Correction & 66 & 88 & 142 & 111 & $2.3 \%$ & $2.6 \%$ & $3.4 \%$ & $2.6 \%$ \\
News Item & 0 & 0 & 0 & 21 & 0 & 0 & 0 & $0.5 \%$ \\
Biographical Item & 1 & 35 & 10 & 15 & $0.0 \%$ & $1.0 \%$ & $0.2 \%$ & $0.4 \%$ \\
Review & 6 & 7 & 6 & 2 & $0.2 \%$ & $0.2 \%$ & $0.1 \%$ & $0.0 \%$ \\
Total & 2,827 & 3,336 & 4,220 & 4,199 & $100.0 \%$ & $100.0 \%$ & $100.0 \%$ & $100.0 \%$ \\
\hline
\end{tabular}

Note. $0.0 \%$ is the result rounded to the nearest tenth.

Journal of Data and Information Science 